\title{
Der Ringversuch - gut und richtig eingesetzt
}

\begin{abstract}
Um Ringversuche zur externen Qualitätskontrolle in der Labormedizin wirksam einzusetzen, müssen optimale Voraussetzungen geschaffen werden. Es braucht Arbeitsanleitungen und Checklisten, damit die Ringversuchsproben korrekt vorbereitet und analysiert werden können. Es braucht aber auch ein grundlegendes Verständnis dafür, dass Qualitätssicherungswerkzeuge nur dann von Nutzen sind, wenn auftretende Fehler als Chance für die Verbesserung von Prozessen wahrgenommen werden. So eingesetzt können Ringversuche nicht nur analytische Probleme, sondern auch grundlegende Probleme im ganzen Ablauf einer Laboranalyse aufdecken. Dank der vielen Teilnehmer können die Qualitätskontrollzentren Erfahrungen sammeln und diese über die Berichte und Kommentare dem ganzen Teilnehmerkollektiv weitergeben. Auf diese Weise, sowie mit speziellen Weiterbildungsprogrammen können die Qualitätskontrollzentren einen Beitrag zur kontinuierlichen Weiterbildung in der Labormedizin leisten.
\end{abstract}

\section{Einleitung}

Ringversuche zur externen Qualitätskontrolle sind ein wichtiges Werkzeug zur Qualitätssicherung in medizinischen Laboratorien.

Bei jedem Ringversuch erhält das teilnehmende Labor von einem Qualitätskontrollzentrum Ringversuchsproben zugeschickt, die analysiert werden müssen. Die Ergebnisse der Analysen werden an das Qualitätskontrollzentrum zurückgeschickt. Dort werden die Daten aller Teilnehmer verglichen, Sollwerte für jede Analyse ermittelt und Statistiken erstellt. Die Teilnehmer erhalten eine Auswertung ihrer Resultate sowie einen allgemeinen Kommentar zum Ringversuch.

Nach Durchführung der externen Qualitätskontrolle erhält das Labor vom Qualitätskontrollzentrum eine Bestätigung, dass die geltenden Anforderungen an die Analytik erfüllt wurden. In der Schweiz werden diese Anforderungen durch die QUALAB festgelegt. Die externe Qualitätskontrolle ist für medizinische Laboratorien wichtig, da normalerweise eine Patientenprobe von einem Labor nur einmal untersucht wird. Die vielen Messwerte der Teilnehmer aus der gleichen Probe, er- möglichen es dem Qualitätskontrollzentrum, dem Teilnehmer ein Feedback über die Qualität der Analytik zu geben. Zusätzlich kann das Zentrum in Form von Summenstatistiken allgemeine Aussagen über die Qualität der Laboratorien und über die verwendeten Methoden machen.

Für die Arbeit der Qualitätskontrollzentren existiert eine spezielle Norm, die ISO 17043 nach der sich diese Organisationen akkreditieren können. Alle drei Schweizer Zentren (BSD-SRK, CSCQ und MQ) sind schon seit langem akkreditiert und werden regelmäßig von der Schweizerischen Akkreditierungsstelle (www.sas.ch) überwacht. Eine spezifische Richtlinie für die optimale Nutzung von Ringversuchen zur Verbesserung der Laboratorien ist beim Clinical and Laboratory Standards Institute (CLSI) erhältlich [1].

\section{Auswahl von Qualitätskontroll- zentren und Anmeldung}

In einigen Ländern gibt es Vorschriften bezüglich den minimalen Anforderungen an die externe Qualitätskontrolle in der Labormedizin. In der Schweiz werden diese von der Schweizerisch- en Kommission für Qualitätssicherung im medizinischen Laboratorium (QUALAB) festgelegt. Die QUALAB überarbeitet jährlich das Dokument „Obligatorische Externe Qualitätskontrolle“, in dem alle Analysen aufgeführt sind, die in der Schweiz zwingend mit Ringversuchen kontrolliert werden müssen. In diesem Dokument wird angegeben, welche Qualitätskontrollzentren für welche Analysen anerkannt sind. Jeder Leistungserbringer der den Krankenversicherern gegenüber Positionen aus der Eidgenössischen Analysenliste (www.bag.admin.ch/al) verrechnet, gilt in der Schweiz als Labor. Unabhängig von der Größe, dem Analysenspektrum und dem Analysenvolumen muss jedes Labor die Regeln der QUALAB einhalten.

Neben den staatlichen Vorgaben gibt es auch Laboratorien, deren Qualitätssicherungssystem auf einer internationalen Norm wie ISO 15189 oder ISO 17025 basiert und durch eine Akkreditierungsstelle (z.B. www.sas.ch) überwacht werden. Solche akkreditierten Laboratorien müssen für alle angebotenen Analysen an Ringversuchen teilnehmen, sofern solche angeboten werden.

Da Laboratorien das Angebot regelmäßig den Bedürfnissen anpassen, muss auch die Anmeldung für die Ringversuche regelmäßig überprüft werden (Tabelle 1). In den meisten Betrieben übernimmt das der Qualitätsbeauftragte. Diese Aufgabe sollte auch in kleinen Betrieben immer der gleiche Mitarbeiter erledigen.

Eine spezielle Situation besteht bei Spitälern, welche viele POCT-Geräte wie Glukosemessgeräte oder Blutgasmessgeräte einsetzen. Diese Geräte befinden sich an unterschiedlichen Standorten und werden von unterschiedlichen Personen bedient. Um den Aufwand für interne Vergleichsmessungen zu reduzieren ist es möglich, alle Geräte für einen Ringversuch 
anzumelden. Das genaue Vorgehen sollte im Voraus mit dem Kontrollzentrum abgesprochen werden.

Falls ein Labor bei Schnelltests keine oder nur die eingebauten Kontrollfelder als interne Kontrolle verwendet, ermöglicht der Ringversuch dem Labor zusätzliche Kontrollmessungen. Dies gilt auch für nicht obligatorische Analysen wie dem Urinteststreifen und dem okkulten Blut im Stuhl.

Qualitätskontrollzentren haben unterschiedliche Teilnehmerkollektive. Falls das Labor aufgrund der staatlichen Bestimmungen bei einem bestimmten Zentrum teilnehmen muss, ist es manchmal von Vorteil, wenn die gleiche Analyse bei einem zweiten Zentrum mit einem passenderen Vergleichskollektiv angemeldet wird. Dies ist meist bei großen Laboratorien der Fall, die sich nicht nur auf lokaler Ebene, sondern auch international mit anderen großen Laboratorien vergleichen möchten.

Jedes Labor hat Analysen, welche sehr oft und solche die sehr selten gemessen werden. Bei Anmeldung der externen Qualitätskontrolle spielt das Analysenvolumen keine Rolle, alle Analysen sollten gleich oft geprüft werden. Falls eine größere Sicherheit gewünscht wird, sollte die Frequenz oder die Zahl der unterschiedlichen Kontrollproben bei der internen Qualitätskontrolle erhöht werden.

Grundlage für eine qualitativ hochwertige Analytik sind gut ausgebildete Mitarbeiter. Es ist deshalb sehr empfehlenswert, Weiterbildungsmöglichkeiten zu nutzen, die von den Zentren angeboten werden. Das sind zum einen die Kommentare, welche mit den Auswertungen der Ringversuche mitgeliefert werden, zum anderen aber auch spezielle Weiterbildungs-Ringversuche wie Präanalytik oder Urinsediment (Abb. 1).

Für die Aus- und Weiterbildung von mikroskopischen Untersuchungen ist es wichtig, das ein Labor eine Präparate-Sammlung aufbaut. Hier bietet die Teilnahme an Blutbild-Ringversuchen

\section{Tabelle 1 Anmeldung von Ringversuchen}

- Gibt es einen Qualitätsbeauftragten, der die Anmeldungen regelmässig überprüft und anpasst?

- Führt das Labor eine Liste mit dem angebotenen Analysenspektrum?

- Welche Geräte werden für welche Analysen verwendet?

- Werden im Betrieb an verschiedenen Orten POCT-Geräte eingesetzt?

- Welche Analysen sind reglementiert? (z. B. durch QUALAB)

- Gibt es Analysen mit zusätzlichem Kontrollbedarf?

- Gibt es reglementierte Analysen bei denen das Vergleichskollektiv der zugelassenen Kontrollzentren nicht optimal ist?

- Besteht Bedarf an regelmässigen Weiterbildungen?

- Soll eine Sammlung von mikroskopischen Präparaten angelegt werden?

\section{MQ 2014-3 U4 Urinsediment}

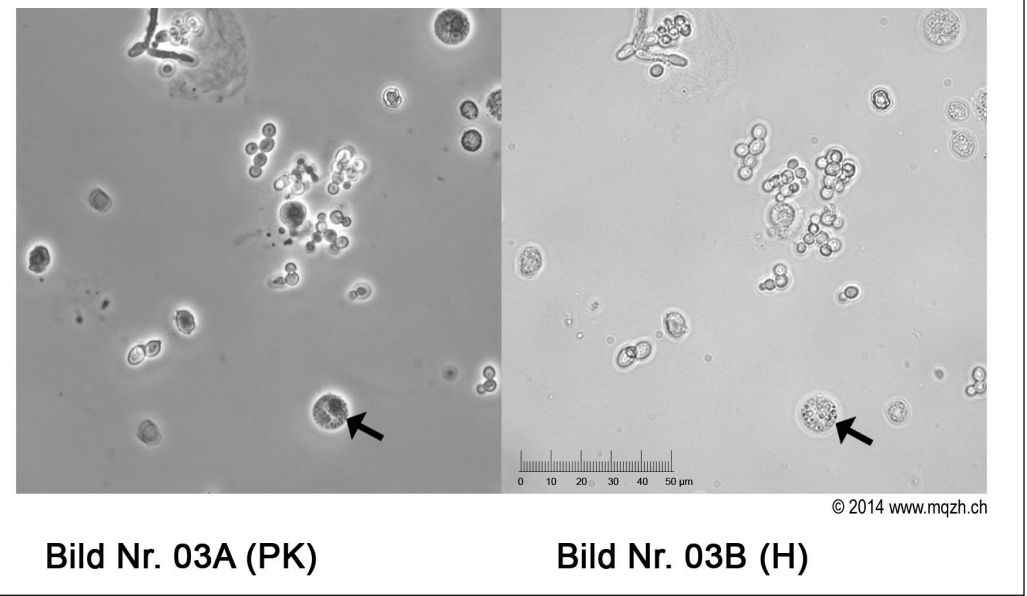

Abbildung 1 Verein für medizinische Qualitätskontrolle (MO): Mikrofotografie (400facher Vergrösserung) vom Urinsediment-Ringversuch. Die Teilnehmer müssen die markierte Zelle im Hellfeld (H) oder im Phasenkontrast (PK) identifizieren. Die Lösung finden Sie in Tabelle 4 am Ende des Artikels

eine gute Möglichkeit, die eigene Sammlung um interessante Fälle zu erweitern. Für Lernende ist eine solche Sammlung von gut charakterisierten Präparaten eine wertvolle Übungsmöglichkeit.

\section{Messen von Ringversuchsproben}

Ringversuchsproben simulieren Patientenproben, und sollten deshalb möglichst wie Patientenproben untersucht werden.

Da Ringversuche nur viermal pro Jahr stattfinden, muss immer damit gerechnet werden, dass sich seit dem letzten
Ringversuch Prozesse im Labor oder beim Qualitätskontrollzentrum verändert haben (Tab. 2). Bei Ankunft der Proben muss deshalb ein Mitarbeiter die Anleitung kontrollieren und überprüfen, ob die erhaltenen Proben und Protokolle noch den aktuellen Analysen und Geräten des Labors entsprechen oder sich inzwischen Änderungen im Labor ergeben haben. Es könnte auch sein, dass aufgrund von ungenügenden Resultaten beim letzten Ringversuch, die Arbeitsanleitung für das Vorbereiten oder das Analysieren der Proben verändert wurde.

In manchen Laboratorien wird die Vorbereitung der Proben durch den Quali- 
Tabelle 2 Messen von Ringversuchsproben

- Gab es im Labor seit dem letzten Ringversuch Veränderungen?

- Gab es im Labor beim letzten Ringversuch Probleme?

- Gibt es Änderungen in der Anleitung des Qualitätskontrollzentrums?

- Ist eine spezielle Probenvorbereitung notwendig?

- Gibt es im Labor ein einheitliches System wie die Ringversuchsproben bezeichnet werden?

- Wird die Ringversuchsprobe von den gleichen Personen und mit den gleichen Geräten wie eine Patientenprobe gemessen?

- Wird die Ringversuchsprobe gleich oft wie eine Patientenprobe gemessen?

- Ist nachvollziehbar wann, von wem und mit welcher Lot-Nummer die Probe gemessen wurde?

- Wer überträgt die Messwerte auf den Protokollbogen?

- Wer kontrolliert den Protokollbogen?

- Wo wird eine Kopie des Protokollbogens abgelegt, bevor dieser ans Zentrum übermittelt wird?

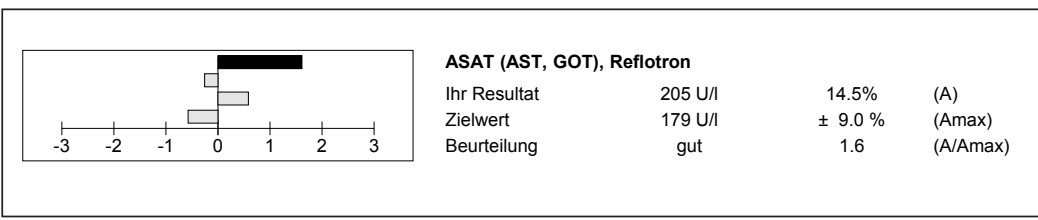

Abbildung 2 Beispiel einer Auswertung des Vereins für medizinische Qualitätskontrolle (MO) für eine quantitative Analyse. In der Grafik wird die relative Abweichung bezüglich der halben OUALAB-Toleranz (A/Amax) dargestellt, wobei der schwarze Balken den aktuellen und die drei grauen Balken die letzten drei Ringversuche darstellen

tätsbeauftragten erledigt. Dabei muss darauf geachtet werden, dass die Proben nach dem, im Labor üblichen System beschriftet werden, damit Probenverwechslungen ausgeschlossen sind. Die Analyse der Ringversuchsproben muss durch den Mitarbeiter gemacht werden, der zu diesem Zeitpunkt auch die Analysen der Patientenproben macht. Mitarbeiter haben manchmal Angst, eine Ringversuchsprobe zu analysieren, weil sie die Verantwortung für das Ringversuchsresultat nicht übernehmen möchten. Dabei sollte man jedoch bedenken, dass die Folgen von Fehlern bei der Analyse von Patientenproben viel schwerwiegender sind.

Messwiederholungen verbessern die Präzision einer Analyse und dürfen bei Ringversuchsproben nur dann gemacht werden, wenn dies aufgrund der geltenden Arbeitsanleitungen im Labor auch bei einer Patientenprobe gemacht würde.
Nach der Analyse der Ringversuchsproben sollte zuerst ein normaler Laborbefund erstellt werden. Dieser wird auf den Protokollbogen des Kontrollzentrums übertragen. Beide Befunde sollten vom Qualitätsverantwortlichen aufbewahrt werden, damit diese be Problemen überprüft werden können. Der Protokollbogen des Ringversuchs unterscheidet sich vom Laborbefund. Bei Analysenbezeichnungen oder Einheiten sollte man sich überlegen, ob es nicht sinnvoll wäre, sich dem Zentrum anzupassen. Zusätzliche Angaben wie die Methodenbezeichnung müssen kontrolliert und wenn nötig ergänzt werden.

Die gleichen Empfehlungen gelten auch für die online-Übermittlung von Resultaten. Dabei muss besonders darauf geachtet werden, dass die eingegebenen Werte kontrolliert und alle $\mathrm{Da}$ ten und Auswertungen im Labor archiviert werden.

\section{Auswertungen der Ringversuche}

Nachdem das Qualitätskontrollzentrum die Resultate der Teilnehmer analysiert und für die verschiedenen Vergleichsgruppen Sollwerte ermittelt hat, schickt es dem Teilnehmer eine Auswertung. Bei quantitativen Analysen enthält diese Auswertung mindestens den Messwert des Teilnehmers, den Sollwert der Vergleichsgruppe, sowie die Beurteilung bezüglich der akzeptablen Toleranz. Meist wird auch der Verlauf der letzten Ringversuche dargestellt, was bei der Beurteilung von Fehlern hilfreich ist (Abb. 2).

Bei Problemen kann man das eigene Ringversuchsresultat mit den Summenstatistiken aller Teilnehmer vergleichen (Abb. 3). Bei einigen Zentren werden solche Statistiken immer mitgeliefert, bei anderen sind die Daten auf der Webseite abrufbar. Solche Berichte können auch weitere Informationen zur Produktion der Proben enthalten:

- die Konzentrationen der zugegebenen Substanzen bei den DrogenurinRingversuchen

- zusätzlich durchgeführte Referenzmethodenwerte beim $\mathrm{HbA1c}$

- Ausdrucke von Hämatologie-Geräten bei den Ringversuchen für das mikroskopische Blutbild.

$\mathrm{Zu}$ den Auswertungen verfasst das Qualitätskontrollzentrum immer einen Kommentar, in dem die wichtigsten Beobachtungen $\mathrm{zu}$ den einzelnen Analysen beschrieben werden. Auch wenn das Labor von einem beschriebenen Problem nicht betroffen ist, lohnt es sich zu überlegen, ob die Prozesse im eigenen Labor so ausgelegt sind, dass dieses Problem sicher nicht auftreten kann. Der Kommentar ist deshalb auch eine Möglichkeit, aus den Fehlern von anderen Teilnehmern zu lernen.

Summenstatistiken die Angaben zu allen Vergleichsgruppen enthalten, können auch für den Vergleich von verschiedenen Analysensystemen verwendet werden. Dabei ist zu beachten, dass nicht alle Ringversuchsproben 


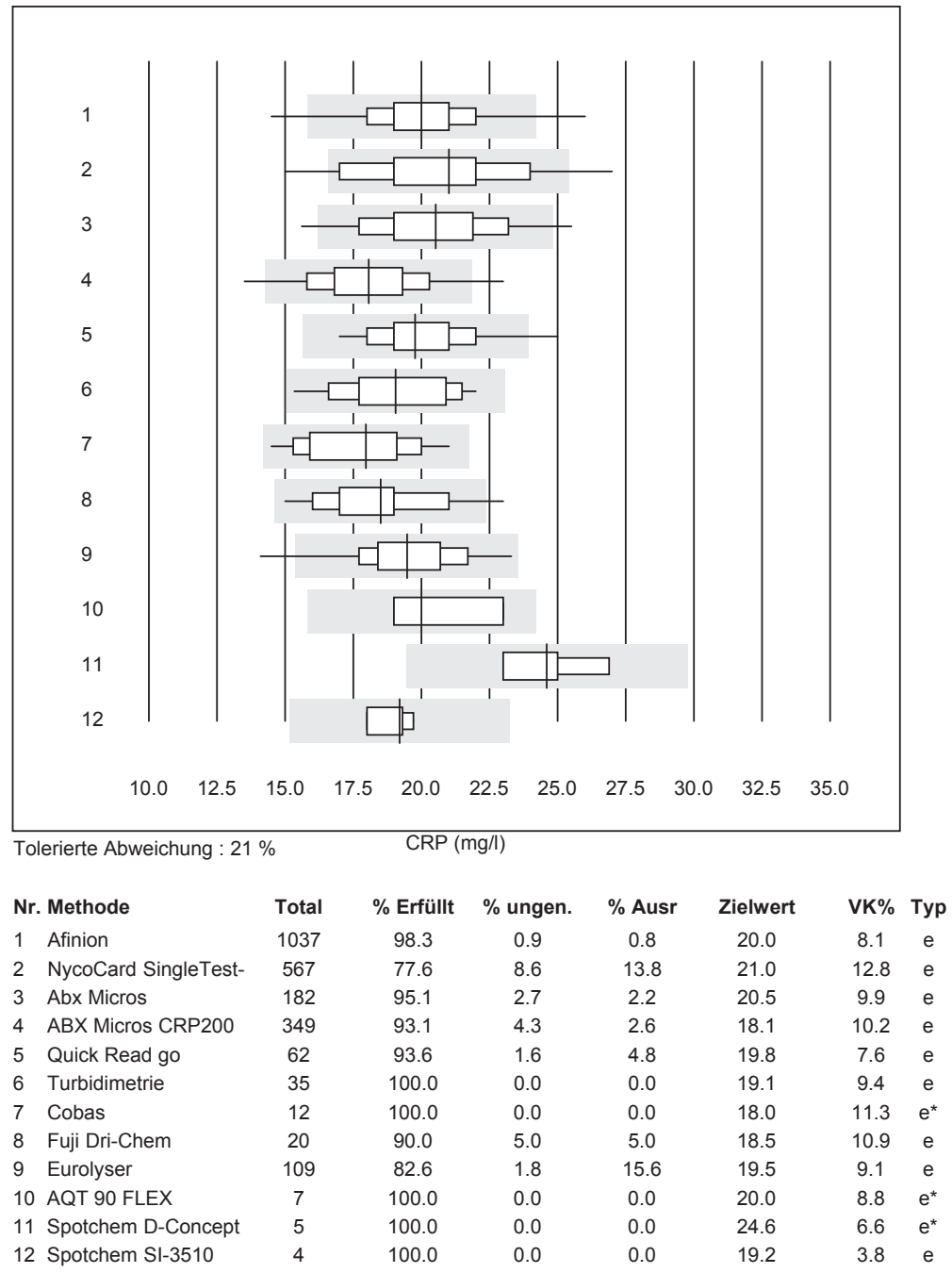

Abbildung 3 Summenstatistiken des Vereins für medizinische Qualitätskontrolle (MO) im Bericht, der nach jedem Ringversuch auf www.mqzh.ch publiziert wird.

Tabelle 3 Abklären von ungenügenden Ringversuchen

- Wurde die richtige Probe bestellt?

- Wurden der Messwert, die Einheit und die verwendete Methode dem Kontrollzentrum korrekt übermittelt?

- Hat das Kontrollzentrum diese Informationen korrekt erfasst?

- War die beobachtete Abweichung medizinisch relevant?

- Ist das Messsystem aufgrund der Leistungsdaten wie Messbereich und Präzision in der Lage die Anforderungen zu erfüllen?

- Existiert eine schriftliche Arbeitsanleitung für die Bedienung des Gerätes und wurde diese eingehalten?

- Erscheinen bei der Analyse im Ausdruck oder auf dem Gerät Warnhinweise?

- Ergibt die Ringversuchsprobe bei einer Nachmessung wieder den gleichen Wert?

- Falls die Abklärungen keine neuen Erkenntnisse ergeben, wird der Vorfall unter „Fehler unbekannter Ursache“ klassiert. gleich gut mit Patientenproben übereinstimmen. Bei manchen Proben treten Matrixeffekte auf, die vom verwendeten Gerät abhängen. Im Zweifelsfalle sollten die Daten mit dem Qualitätskontrollzentrum besprochen werden.

\section{Abklärung von ungenügenden Resultaten}

Alle Laboratorien erhalten manchmal Auswertungen mit ungenügenden Resultaten. Das ist keine Schande, sondern eine Chance die eigenen Prozesse zu überprüfen und zu verbessern. Deshalb muss jedes ungenügende Resultat sorgfältig abgeklärt werden.

Man unterscheidet zwischen Fehlern, die aufgrund der speziellen Prozesse für den Ringversuch auftreten und Fehlern, die auch bei Patientenproben auftreten können.

Typische Ringversuchsfehler hängen mit der speziellen Vorbereitung und der Beschaffenheit der Ringversuchsproben sowie mit dem speziellen Berichtformat, dem Protokollbogen zusammen. Auch wenn diese Fehler nicht bei Patienten auftreten können, ist es trotzdem möglich, dass diese Fehler auf grundlegende Probleme wie die Ausbildung der Mitarbeiter, unterschiedliche Analysenbezeichnungen oder veraltete Einheiten hinweisen. Die Abbildungen 4-6 zeigen solche Fehler. Analytische Fehler können auftreten weil das Gerät nicht korrekt bedient wird oder weil das Gerät oder die Reagenzien defekt sind. Das Mischproblem bei Abbildung 7 ist ein Grenzfall, da es Laboratorien gibt, die praktisch ausschliesslich Kapillarblut untersuchen. Die defekten Teststreifen bei Abbildung 8 haben jedoch direkte Auswirkungen auf die Analyse von Patientenproben.

\section{Abweichungsbericht}

Ein Abweichungsbericht sollte so verfasst sein, dass ein Experte der nicht im 


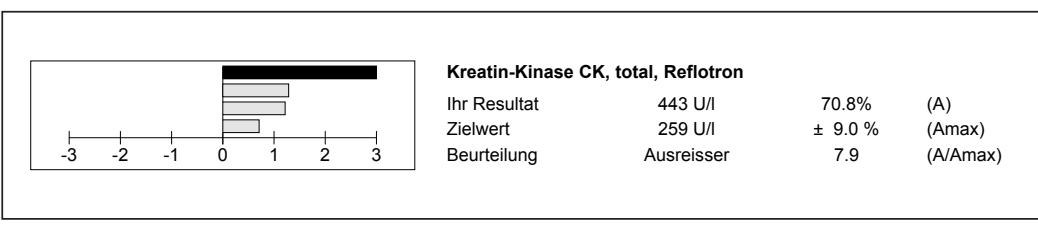

Abbildung 4 MQ-Auswertung: Der Teilnehmer hat beim Übertragen der Resultate den Kreatinin-Messwert auf dem Protokoll unter „Kreatin-Kinase“ eingetragen

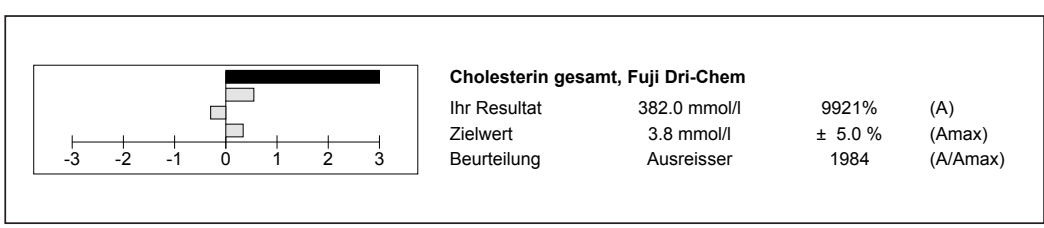

Abbildung 5 MQ-Auswertung: Der Teilnehmer hat beim Übertragen des Cholesterin-Resultates das Komma vergessen

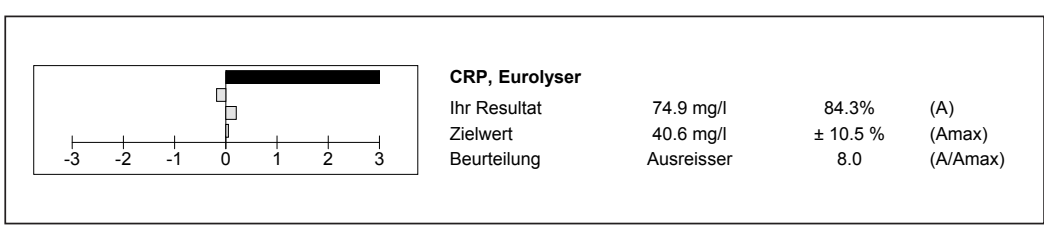

Abbildung 6 MQ-Auswertung: Der Teilnehmer misst normalerweise nur CRP aus Vollblut. Er hat nicht beachtet, dass die Ringversuchsprobe Plasma enthält und das Gerät deshalb umgestellt werden muss

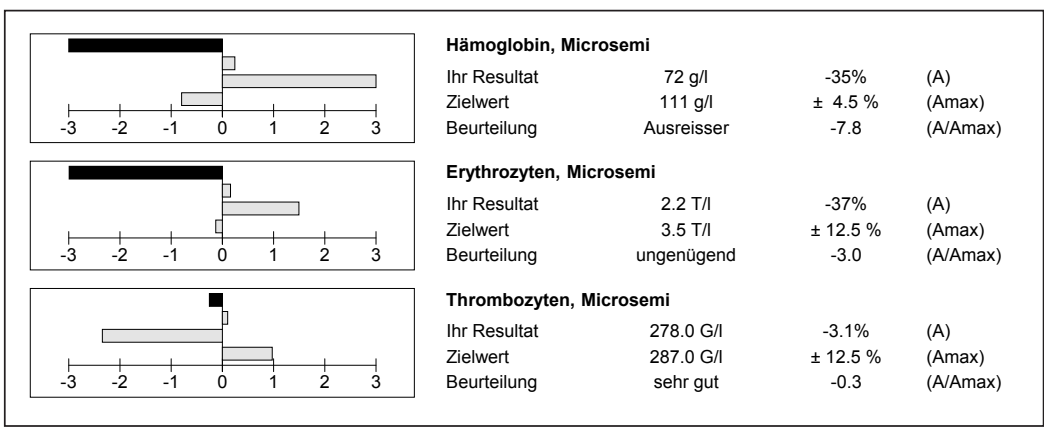

Abbildung 7 MQ-Auswertung: Der Teilnehmer hat die Hämatologie-Probe nicht gut gemischt

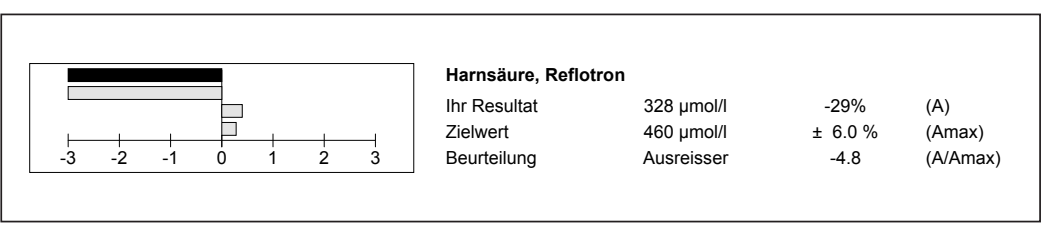

Abbildung 8 MO-Auswertung: Der Teilnehmer verwendet falsch gelagerte oder abgelaufene Teststreifen
Betrieb arbeitet bei einem externen $\mathrm{Au}$ dit oder im Rahmen einer Supervision nachvollziehen kann, welche Art von Fehler aufgetreten ist, welche Abklärungen gemacht wurden und wie weit Korrekturmaßnahmen ergriffen wurden. Damit das Labor Probleme möglichst effizient aufdecken und beheben kann, sollte es bei einem ungenügenden Ringversuch möglichst systematisch den ganzen Ablauf überprüfen und alle $\mathrm{Maßnahmen}$ und Informationen im Abweichungsbericht festhalten.

Der Abweichungsbericht sollte in einem ersten Teil den beobachteten Fehler möglichst genau beschreiben. Dazu gehören auch alle verfügbaren Informationen über die fehlerhafte Ringversuchsanalyse.

Im zweiten Teil wird überprüft, ob alle Daten korrekt übermittelt, erfasst und ausgewertet wurden oder ob Übertragungsfehler oder Erfassungsfehler aufgetreten sind.

Falls keine administrativen Fehler aufgetreten sind, wird in einem dritten Teil die Analytik untersucht. Dazu gehört eine Analyse der internen Qualitätskontrolldaten aus dem Zeitraum des Ringversuches, oder eine Nachmessung der Ringversuchsproben. Diese lassen sich in der Regel beim Kontrollzentrum nachbestellen.

Wird die Analytik aufgrund der Fehleranalyse verändert, muss überprüft werden, ob die Messwerte nun besser mit dem Sollwert übereinstimmen. Diese Maßnahmen sowie die Bestätigung, dass wieder alles unter Kontrolle ist, werden in einem vierten Teil dokumentiert.

In bis zu $25 \%$ der Fälle liefern die Abklärungen keine klare Ursache und es können keine Korrekturmaßnahmen ergriffen werden. Falls ein Fehler zum ersten Mal auftritt, und man alle üblichen Ursachen ausgeschlossen hat, schließt man den Bericht mit dem Befund „unbekannter Fehler“ ab.

Tritt der Fehler wiederholt auf, muss das Labor externe Hilfe beim Lieferanten, bei Beratern oder beim Qualitätskontrollzentrum suchen. 
Tabelle 4 Lösung zu Abbildung 1, Resultate der Teilnehmer

\begin{tabular}{|l|c|}
\hline $\begin{array}{l}\text { Angabe der } \\
\text { Teilnehmer }\end{array}$ & Anzahl \\
\hline Leukozyten & 263 (richtig) \\
\hline Rundepithelien & 18 \\
\hline Nierenepithelien & 16 \\
\hline Lipide & 6 \\
\hline $\begin{array}{l}\text { Epithelien } \\
\text { (andere als Platten-) }\end{array}$ & 3 \\
\hline
\end{tabular}

Die Auswertungen der Ringversuche wie auch die Abweichungsberichte müssen mindestens fünf Jahre aufbewahrt werden.

\section{Kontinuierlicher \\ Verbesserungsprozess}

Wenn bei einem ungenügenden Ringversuchsresultat eine Ursache für den Fehler gefunden wurde, muss sich das Labor überlegen, ob nicht ein grundsätzliches Problem vorliegt, das diesen Fehler zulässt. Wenn beispielsweise unübliche Analysenbezeichnungen verwendet werden, die beim Ausfüllen des Protokollbogens zuerst übersetzt werden müssen, dann kann das immer wieder zu Verwechslungen führen. Eine nachhaltige Lösung wäre, die Bezeichnungen zu ändern.

Bei technischen Problemen mit einem alten Gerät kann kurzfristig helfen, alle Mitarbeiter zu schulen. Vielleicht ist es aber sinnvoller, ein neues Gerät anzuschaffen, das über weniger Bedienungsschritte verfügt und präziser arbeitet.

\section{Making best use of external quality} assessment

To receive a maximum benefit from external quality assessment, the laboratory has to fulfill certain requirements. There have to be standard operating procedures and checklists for the correct sample processing and analysis. It is equally important, that the staff has a basic understanding how this quality-tools work and that detected errors are used as a chance to improve the processes within the laboratory. The benefit of surveys for external quality assessment is not only limited to the analytical phase, but also to some aspects of the pre and postanalytical processes. Due to the many participants, the survey providers are able to collect a lot of practical knowledge. All participants can learn from this by reading the survey reports and commentaries. With this, and with special educational surveys, the providers of surveys are able to offer an opportunity of continuous education to the laboratories.

\section{Literatur}

1. Clinical and Laboratory Standards Institute. Using Proficiency Testing to Improve the Clincial Laboratory; Approved Guideline-Second Edition. CLSI document GP27-A2.

Links

I. Blutspendedienst Bern, www.bsdbe.ch/ringversuchszentrum

II. Schweizerisches Zentrum für Qualitätskontrolle, Genf, www.cscq.ch

III. Verein für medizinische Qualitätskontrolle, Zürich, www.mqzh.c.

IV. Eine Liste mit den Europäischen Qualitätskontrollzentren führt die EQALM, www.eqalm.org

V. Schweizerische Kommission für Qualitätssicherung im medizinischen Laboratorium, Bern, www. qualab.ch

\section{Korrespondenzadresse}

Dr. phil. Roman Fried

Verein für medizinische

Qualitätskontrolle

Institut für Klinische Chemie

Universitätsspital Zürich

8091 Zürich

roman.fried@usz.ch 